\title{
Erude du ruissellement sur un plan incliné par vélocimètrie polarographique
}

\author{
Daniel Campergue* el Gérard Cogner**
}

\section{Introduction}

Le ruissellement en film mince d'un liquide le long d'une paroi solide revêt une grande importance pratique, qu'il soit d'origine naturelle ou réalisé dans des installations de type industriel. Une connaissance approfondie de l'hydrodynamique d'un tel écoulement est essentielle pour une meilleure compréhension des phénomènes d'érosion, de transfert de chaleur et de masse dont il est généralement le siège.

L'ensemble des résultats, tant théoriques qu'expérimentaux, publiés sur le sujet avant 1964, a été remarquablement répertorié par Fulford [1]. Les recherches récentes se rapportent plus particulièrement à l'étude de la stabilité du régime laminaire lisse et à la caractérisation des régimes d'onde et turbulent. L'écoulement est le plus souvent étudié le long d'un tube vertical [2] ou sur un plan soit vertical [3], soit faiblement incliné par rapport à l'horizontale [4]. Le présent article montre comment, disposant d'une technique de mesure, la polarographie électrochimique, permettant la détermination locale et instantanée du gradient pariétal de vitesse, il a été possible de mener une étude systématique du ruissellement plan pour toutes les inclinaisons et aux différents régimes d'écoulement.

(*) Laboratoire de Cinétique Physique et d'Electrochimie C.U. de Perpignan.

(**) L.E.M.T.A.E.N.S.E.M., Institut National Polytechnique de Lorraine, Nancy.

\section{Conditions expérimentales}

1. - Principe de la méthode de mesure

La technique utilisée : "la vélocimétrie polarographique" est l'équivalent en transfert de masse de l'anémométrie à fil (ou à film) chaud à température constante [5] ; son principe, brièvement rappelé, est le suivant :

Le fluide en écoulement est une solution diluée d'un couple oxydant- réducteur défini par l'équilibre :

$$
\mathrm{Ox}+n e \stackrel{\leftarrow}{\rightarrow} \operatorname{Red}
$$

en présence d'un électrolyte support en large excès.

L'élément de mesure est une électrode de petite dimension ; lorsqu'elle est portée à un potentiel suffisamment cathodique, dans le cas d'une réaction électrochimique rapide, elle est le siège d'un courant de réduction contrôlé par la diffusion convective entre le sein de l'écoulement où la concentration des ions actifs est constante et l'électrode où leur concentration est nulle. Le courant limite est alors fonction du champ de vitesse au voisinage de la surface réagissante. Pour une électrode rectangulaire affleurant la paroi et placée perpendiculairement à la direction $\vec{x}$ de l'écoulement, la couche limite de concentration a une épaisseur $\delta_{c}$ très faible [6]; dans l'hypothèse d'un régime permanent, la vitesse dans cette zone peut être représentée par $U=S y$ ( $y$ distance normale au plan). L'équation du bilan de matière s'écrit dans ce cas :

$$
S y \frac{\partial c}{\partial x}=D \frac{\delta^{2} c}{\partial y^{2}}
$$


avec les conditions aux limites

$$
\begin{gathered}
x=0, y>0: C=C_{0} \\
x>0, y=0: C=0 \\
y \rightarrow \infty: C \rightarrow C_{0} .
\end{gathered}
$$

Le courant limite mesuré à l'électrode rectangulaire est alors donné par la relation:

$$
J=\left.n F \int_{\mathrm{A}} D \frac{\partial c}{\partial y}\right|_{y=0} d A=n F C_{0} A \cdot 0,807\left(D^{2} S / L\right)^{1 / 3}
$$

valable pour $\left(L^{2} S / D\right)>5000$

$n$ est le nombre d'électrons mis en jeu dans la réaction électrochimique, $F$ le nombre de Faraday, $A$ la surface de l'électrode, $L$ sa largeur, $D$ le coefficient de diffusion de l'ion actif en concentration $C_{0}$.

Dans le cas d'une électrode circulaire de diamètre $d_{0}$, souvent utilisée en pratique, on définit une largeur équivalente $L_{e}=0,82 d_{0}$. On déduit de (2) le frottement pariétal :

$$
\tau=\mu S=\frac{\mu L}{D^{2}}\left(\frac{J}{0,807 n F C_{0} A}\right)^{3}
$$

$\mu$ étant la viscosité dynamique du fluide supposé newtonien.

Pour un écoulement non permanent, tant que les fluctuations de vitesse ne sont pas de fréquence et d'amplitude trop élevées, on peut faire l'approximation de l'état quasi-stationnaire.

$$
\frac{s}{\bar{S}}=\frac{3 j}{\bar{J}}
$$

$s$ et $j$ représentant les fluctuations du gradient pariétal de vitesse et du courant autour de leurs moyennes respectives $\bar{S}$ et $\bar{J}$. Dans cette hypothèse, la constante de temps de la sonde s'écrit [8]:

$$
\chi=0,911\left(\frac{L^{2}}{\overline{D S}^{2}}\right)^{1 / 3}
$$

\section{2. - Dispositif d'étude}

Le schéma de l'installation est présenté sur la figure 1a et $b$ (un réservoir $A$ thermostaté à niveau constant alimente uniformément le plan d'étude en plexiglas de 70 centimètres de longueur, $19 \mathrm{~cm}$ de largeur et pouvant prendre toutes les inclinaisons $\theta$ entre $0^{\circ}$ et $90^{\circ}$ ). Au bas du plan, deux parois minces biseautées distantes de $8 \mathrm{~cm}$ permettent d'isoler le liquide s'écoulant dans la région centrale, éliminant ainsi, lors de la mesure de $Q$ débit par unité de largeur, les effet de freinage [9] et de capillarité [10] dûs aux parois latérales.

Le liquide repris dans le réservoir $B$ est remonté en $A$ par une pompe péristatique. L'ensemble du circuit est réalisé en matériaux isolants électriques et chimiquement inertes vis-àvis des solutions utilisées solutions d'iode dans de l'eau $\left(C_{0}=10^{-6} \mathrm{~mole} / \mathrm{cm}^{3}\right)$

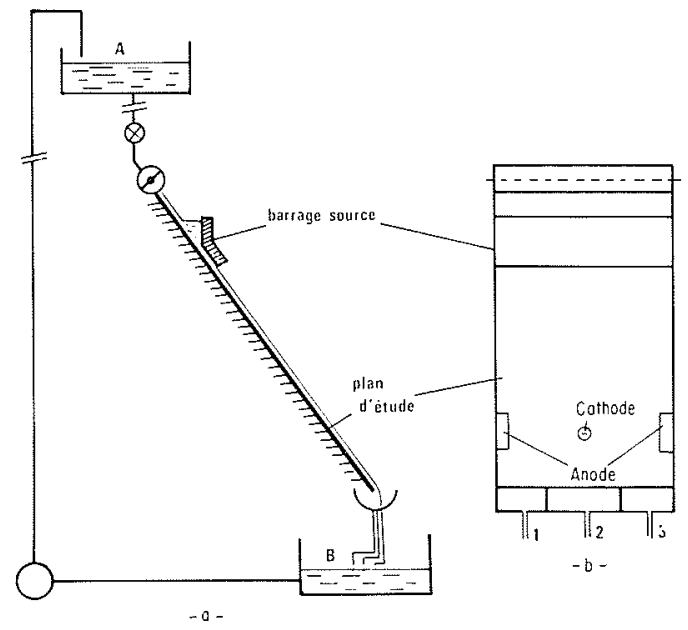

Figure 1. - Schéma de l'installation

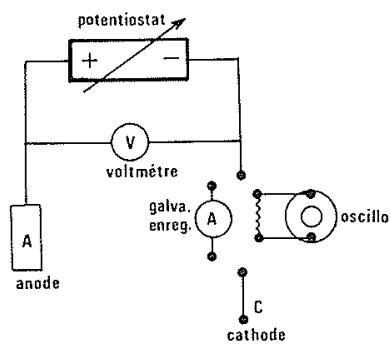

Figure 2. - Circuit électrique

avec un excès d'iodure de potassium $\left(10^{-3} \mathrm{~mole} / \mathrm{cm}^{3}\right)$. L'équilibre oxydant-réducteur s'écrit : $I_{3}^{-}+2 e^{-} \rightleftharpoons 3 I^{-}$. A $20^{\circ} \mathrm{C}$, température des expériences, le coefficient de diffusion de l'ion actif $I_{3}^{-}$est : $D=0,985 \times 10^{-5} \mathrm{~cm}^{2} / \mathrm{s}$, la viscosité dynamique $\mu=0,968 \times 10^{-2}$ poise et la viscosité cinématique $\nu=0,958 \times 10^{-2} \mathrm{~cm}^{2} / \mathrm{s}[11]$

Les électrodes test (cathodes) en platine, l'une rectangulaire, perpendiculaire à l'écoulement $(l \times \mathrm{L}=9 \mathrm{~mm} \times$ $0,3 \mathrm{~mm})$, l'autre circulaire $\left(d_{0}=0,4 \mathrm{~mm}\right)$, sont montées sur des supports interchangeables affleurant la surface du plan, à $50 \mathrm{~cm}$ en aval de la source, le régime d'écoulement étant alors complétement établi $[12,13]$. La contreélectrode (anode) est constituée de deux plaques de nickel de grande surface noyées dans le plan et situées de part et d'autre de la cathode. Le circuit électrique présenté sur la figure 2 comprend une source de tension permettant de maintenir le potentiel de l'électrode test sur le palier de la courbe courant-tension correspondant à la réduction de $I_{3}^{-}$. Les mesures sont faites sur un galvanomètre enregistreur pour les valeurs moyennes des courants limites, sur un oscilloscope pour la caractérisation de leurs fluctuations.

\section{Résultats}

Les dimensions des électrodes et les conditions de l'écoulement sont telles que l'approximation de l'état quasi-stationnaire peut être retenue vis-à-vis des résultats obtenus. Ainsi, les relations (3) et (4) sont utilisées pour déterminer les valeurs moyennes et les fluctuations du frottement à partir des valeurs moyennes et des fluctuations du courant limite. 


\section{1. - Valeurs moyennes du frottement}

Les figures 3 et 4 représentent le frottement moyen respectivement en fonction du nombre de Reynolds $\mathrm{du}$ ruissellement $\left(R e=\frac{Q}{\nu}\right)$ et de l'inclinaison du plan. D'après les pentes des courbes, en coordonnées logarithmiques, on constate que :

$$
\tau \sim R e^{a}(\sin \theta)^{b}
$$

avec $0,33<a<0,55$ et $0,66<b<0,85$

pour $20<\operatorname{Re}<1100$ et $0^{\circ}<\theta<90^{\circ}$

On remarque qu'aux faibles inclinaisons et faibles débits, les résultats sont en accord avec la théorie de Nusselt [14] en régime laminaire :

$$
\tau=\left(3 \rho^{2} g^{2} \mu Q \sin ^{2} \theta\right)^{1 / 3}=\left(3 \rho^{3} g^{2} \nu^{2} \operatorname{Re} \sin ^{2} \theta\right)^{1 / 3}
$$

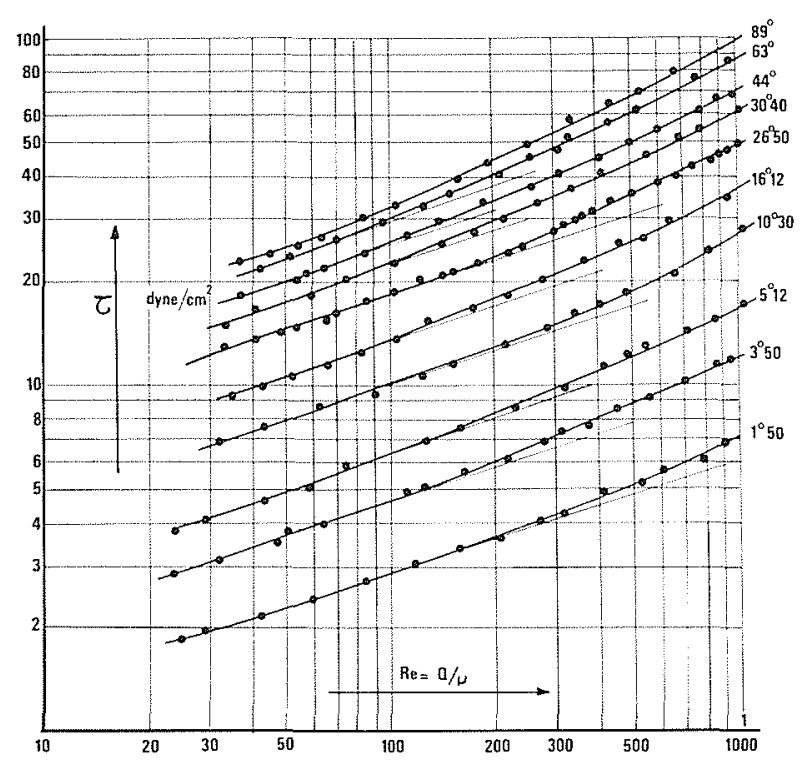

Figure 3. - Frottement pariétal moyen fonction du nombre de Reynolds

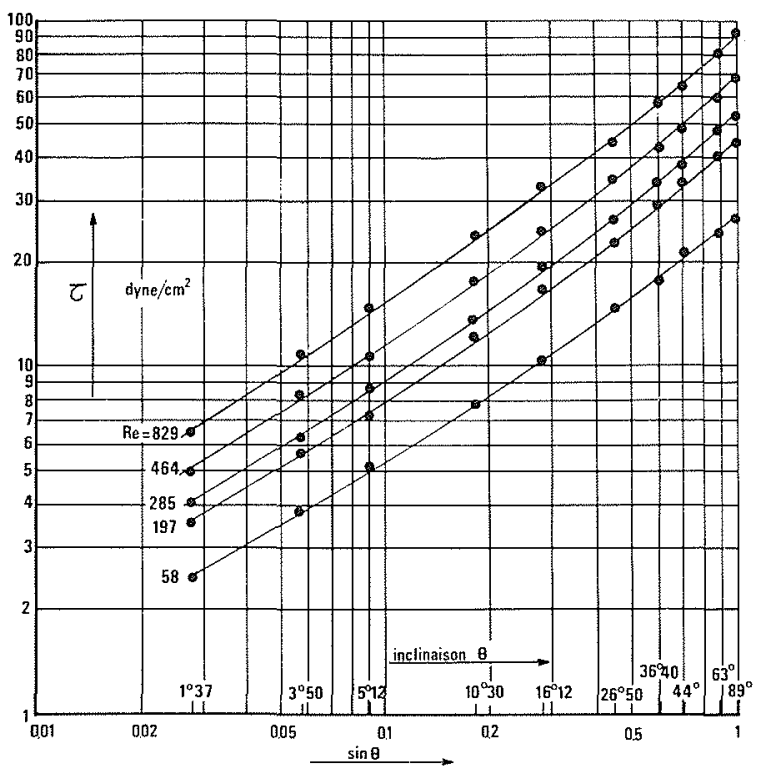

Figure 4. - Frottement pariétal moyen fonction de l'inclinaison avec $\rho$ masse volumique du fluide et $g$ accélération de la pesanteur. Ils s'en écartent d'autant plus aux grands débits que l'inclinaison est plus forte. C'est ainsi que la loi $\tau \sim R e^{1 / 3}$ n'est plus respectée au-delà de :

$$
\begin{aligned}
& R e=300 \text { pour } \theta=1^{\circ} 50^{\prime} \\
& R e=200 \text { pour } \theta=10^{\circ} 30^{\prime} \\
& R e=90 \text { pour } \theta=89^{\circ} .
\end{aligned}
$$

Ceci peut s'interpréter par le fait que la transition du régime laminaire lisse au régime d'onde et au régime turbulent se fait d'autant plus sentir à la paroi que le film est mince, donc, à débit constant, que le plan est incliné.

\section{2. - Epaisseur moyenne du film}

La mesure de l'épaisseur du film ruisselant est importante pour l'évaluation des transferts de chaleur et de masse dont il peut être le siège.

En régime laminaire lisse établi, le frottement qui s'exerce sur la surface est négligeable et le bilan des forces qui agissent sur une longueur unité de film donne :

$$
h=\tau /(\rho g \sin \theta)
$$

On suppose qu'en régime d'onde et turbulent établis, la relation (7) est encore vérifiée en moyenne. Ainsi, à partir des valeurs expérimentales du frottement moyen, il est possible de déterminer l'épaisseur moyenne du film.

La figure 5 représente les variations de l'épaisseur du film en fonction du nombre de Reynolds pour les trois inclinaisons $5^{\circ} 12^{\prime}, 26^{\circ} 50^{\prime}$ et $89^{\circ}$. Les résultats sont comparés aux courbes théoriques correspondant au profil de vitesse semi-parabolique de Nusselt en régime laminaire :

$$
h=[3 Q v /(g \sin \theta)]^{1 / 3}=\left[3 v^{2} \operatorname{Re} /(g \sin \theta)\right]^{1 / 3}
$$

et au profil universel de vitesse (P.U.V.), type Nikuradse, en régime turbulent :

$$
\begin{aligned}
& \text { (9) }\left\{\begin{array}{llc}
u^{+}=y^{+} & \text {pour } & 0 \leqslant y^{+} \leqslant 5 \\
u^{+}=-3,05+5 \log y^{+} & \text {pour } & 5<y^{+} \leqslant 30 \\
u^{+}=5,5+2,5 \log y^{+} & \text {pour } & 30<y^{+} \leqslant h^{+}
\end{array}\right. \\
& \operatorname{avec} u^{+}=u / u^{*} \\
& y^{+}=y u^{*} / \nu \text { et } h^{+}=h u^{*} / \nu \text { distance à la paroi et } \\
& u^{*}=(\tau / \rho)^{1 / 2} \quad \text { vitesse de frottement }
\end{aligned}
$$

Dans ce modèle, l'écoulement ne peut être considéré comme turbulent que lorsque $h^{+}>30$. En intégrant ces vitesses sur toute l'épaisseur du film, on obtient [15] :

$$
\begin{aligned}
& R e=u^{+} h^{+}=h^{+}\left(3,0+2,5 \log h^{+}\right)-64 \\
& h^{+}=(g \sin \theta)^{1 / 2} \cdot h^{3 / 2} / \nu
\end{aligned}
$$

Les relations (10) et (11) permettent de déterminer $h$ connaissant le débit et l'inclinaison du plan. 


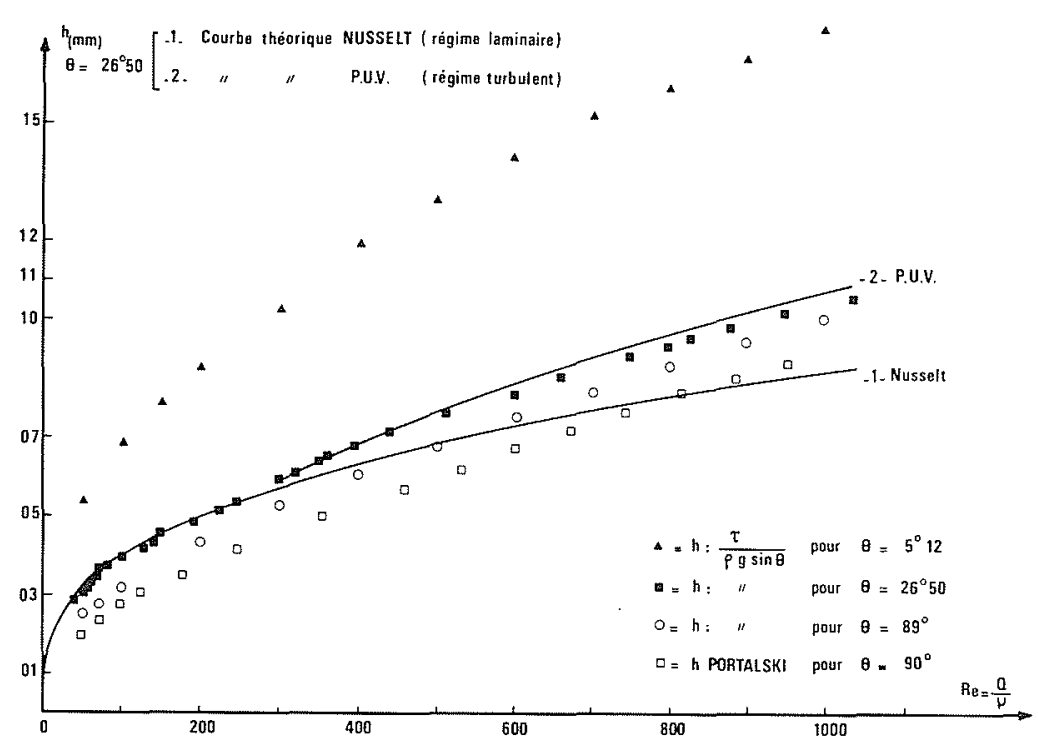

Figure 5. - Epaisseur du film fonction du nombre de Reynolds

Pour l'inclinaison $\theta=26^{\circ} 50^{\prime}$ qui a été retenue à titre de comparaison, la courbe du régime laminaire représente correctement les résultats expérimentaux. jusqu'à $R e \simeq 275$; on observe pour $50<R e<150$ des valeurs légèrement inférieures correspondant au régime d'onde pour lequel Kapitsa [16] puis Levich [17] ont prévu une diminution de $h$ par rapport à la relation (8) de l'odre de $7 \%$. Au-delà et jusqu'à $R e=1100$, limite de nos expériences, la courbe du régime turbulent obtenue à partir de (10) et (11) convient à mieux que $5 \%$ près. L'écart de $15 \%$ environ constaté avec les résultats de Portalski [3] dans le cas d'un plan vertical peut s'expliquer par la différence des techniques expérimentales utilisées (drainage et mesure de capacité électrique du film pour l'exemple cité).

\section{3. - Coefficient de frottement}

Si la relation (7) est justifiée, le coefficient de frottement $C_{f}=\tau /\left(\rho U_{m}^{2} / 2\right)$ s'écrit :

$$
C_{f}=\frac{2 g h \sin \theta}{U_{m}^{2}}=\frac{2 \sin \theta}{F r^{2}}=\frac{2 g h^{3} \sin \theta}{Q^{2}}=\frac{2 \mathrm{~N}}{R e}
$$

avec $F r=\frac{U_{m}}{\sqrt{g h}}$ nombre de Froude, $N=\frac{R e \sin \theta}{F r^{2}}$ nombre adimensionnel caractérisant l'écoulement, $U_{m}$ étant la vitesse moyenne dans le film. Il peut, dans ce cas, être interprété comme une épaisseur adimensionnelle [1] et à ce titre présente un intérêt particulier.

Le réseau de courbes de la figure 6 met en évidence la loi de variation de $C_{f}$ en fonction du nombre de Reynolds et de l'inclinaison du plan.

- Pour de faibles débits, et quelle que soit l'inclinaison du plan, le coefficient de frottement suit la loi de Nusselt

$$
N=3 \quad \text { soit } \quad C_{f}=6 / R e
$$

Il s'en écarte d'autant plus vite que l'inclinaison est forte : pour $R e \simeq 60$, lorsque $\theta=89^{\circ}$, et pour $R e \simeq 250$ lorsque $\theta=5^{\circ} 12^{\prime}$.

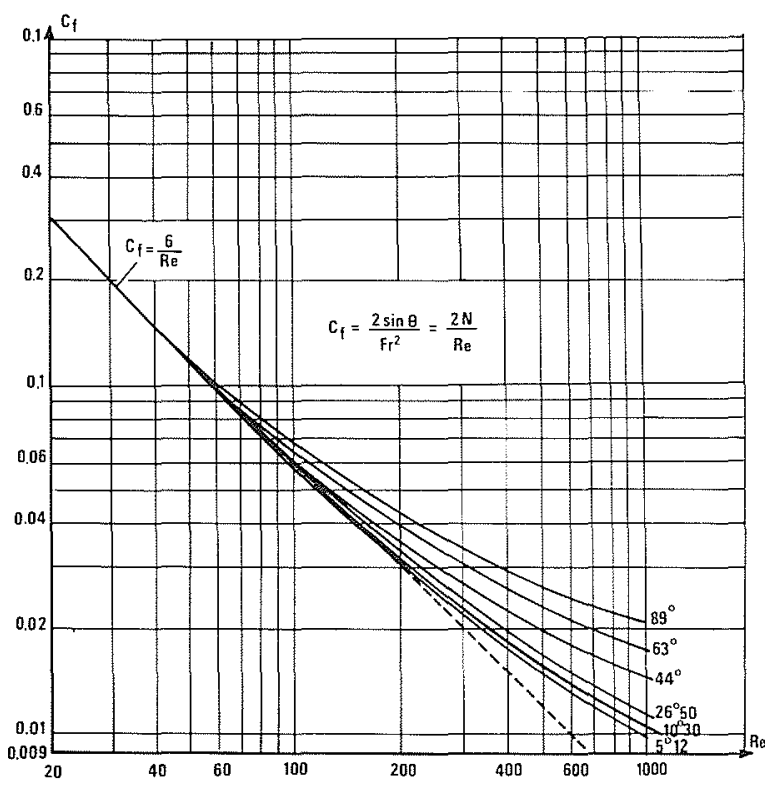

Figure 6. - Coefficient de frottement fonction du nombre de Reynolds à différentes inclinaisons

- La pente des courbes décroît lorsqu'on augmente l'inclinaison et le débit ; ainsi, pour $R e=1000$ sur un plan vertical : $C_{f} \sim R e^{-0,35}$

On peut noter que les résultats disponibles dans la littérature essentiellement dans le cas d'un plan ou d'un tube vertical laissent prévoir une pente -1 jusqu'à $R e=300-400$, c'est-à-dire au-delà de la limite observée dans nos expériences, et une pente variant de $-1 / 2$ à $-1 / 4$ pour $\operatorname{Re}>400$ [1].

\section{4. - Fluctuation du frottement en régime d'onde}

Très commun de façon naturelle, le régime d'onde a des applications industrielles multiples ; du fait de l'accroissement de l'aire interfaciale qu'il représente et du renouvellement de celle-ci, son efficacité pour le transfert de chaleur et de masse se trouve accrue 
par rapport au régime laminaire lisse. D'après la relation (5), les fluctuations de courant enregistrées au niveau de l'électrode de mesure permettent de déterminer les fluctuations du gradient pariétal de vitesse, et donc du frottement, provoquées par le passage d'une onde. Un exemple de photographies prises à l'oscilloscope est présenté sur la figure 7 pour l'inclinaison $\theta=16^{\circ} 12^{\prime}$ et deux débits : $R e=54$ et $R e=155$. On peut définir une amplitude et une période moyennes malgré la distorsion des ondes observées pratiquement dès leur naissance avec la formation de rides sur leur front aval.

\section{1. - Amplitudes $\tau^{\prime}$ des fluctuations du frottement}

$J^{\prime}$ étant l'amplitude des fluctuations de courant, à mieux que $1 \%$, on peut écrire :

$$
\tau^{\prime}=\frac{\mu}{D^{2}} \frac{L}{\left(0,807 n F C_{0} A\right)^{3}} 3 \cdot \bar{J} . J^{\prime}
$$

Les courbes de la figure 8 représentent les amplitudes des fluctuations de frottement en fonction du nombre de Reynolds pour une série d'inclinaisons du plan. Les fluctuations sont enregistrées dès l'apparition du régime d'ondes constatée par observation directe du film pour $R e=20-30$. Quelle que soit l'inclinaison, elles passent par un maximum pour $R e=80-100$, puis décroissent pour céder la place graduellement au régime turbulent pour $R e=300-500$. Ainsi, les rides initialement bidimensionnelles prennent un caractère progressivement tridimensionnel puis de plus en plus aléatoire. les fluctuations sont d'autant plus marquée que le plan est incliné.

Les valeurs relatives $\tau^{\prime} / \tau$ sont reportées sur la figure

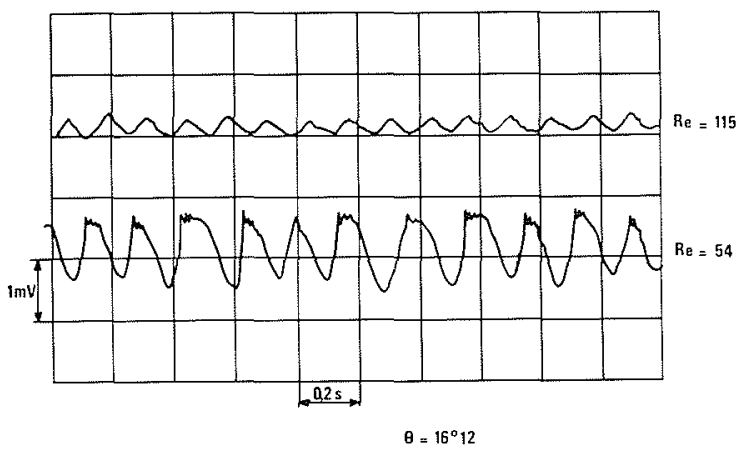

Figure 7. - Enregistrements des fluctuations de courant pour $\theta=16^{\circ} 12^{\prime} \operatorname{Re}=54$ et $\operatorname{Re}=155$

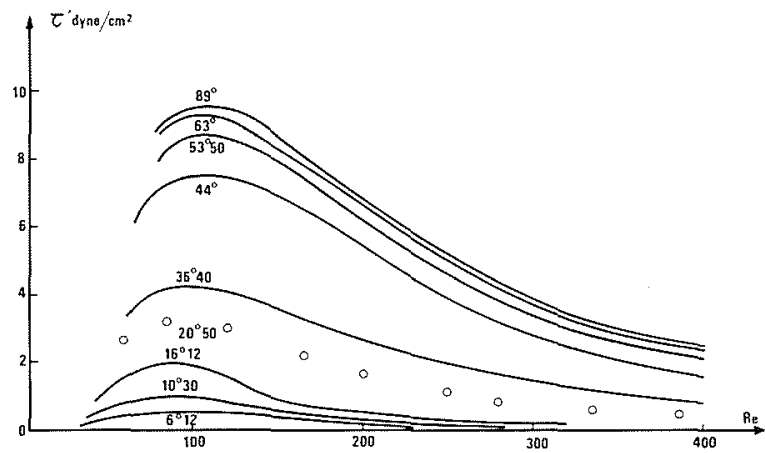

Figure 8. - Fluctuations du frottement fonction de $R e$ à différentes inclinaisons
9. On constate que le rapport peut atteindre $25-30 \%$ pour $R e=80-100$ lorsque le plan est vertical ; il est pratiquement proportionnel à $(\sin \theta)^{2 / 3}$

Ces résultats peuvent être comparés à ceux obtenus :

- Expérimentalement par Wragg et Einarsson [2]. A l'aide d'une technique électrochimique semblable, le long d'un tube vertical, à $50 \mathrm{~cm}$ de la source pour $R e=100$, il trouve une amplitude relative du transfert de matière de l'ordre de $3,5 \%$, ce qui correspond à $10,5 \%$ pour le frottement

- Théoriquement

par Levich [17] qui propose une expression de l'épaisseur $h$ du film de la forme $h=\bar{h}(1+\psi)$ avec $\psi=\alpha \sin (k x-\omega t)$ variation relative de l'épaisseur. Une approche numérique pour un plan vertical donne $\alpha=0.21$

et par Gjevik [18] qui a calculé numériquement le rapport $h^{\prime} / \bar{h}=0,35$.

On remarque que les valeurs données ci-dessus concernent les variations d'épaisseur de film. La relation (7) est valable en moyenne mais inapplicable pour relier à chaque instant les fluctuations de $h$ aux fluctuations de $\tau$. On peut également considérer avec Brock [19] que l'onde est périodique mais non sinusoidale avec un front beaucoup plus raide vers l'aval que vers l'amont (certains types d'enregistrement pourraient être interprétés dans ce sens).

\section{2. - Fréquence des ondes}

On constate sur la figure 10 , où sont reportés les

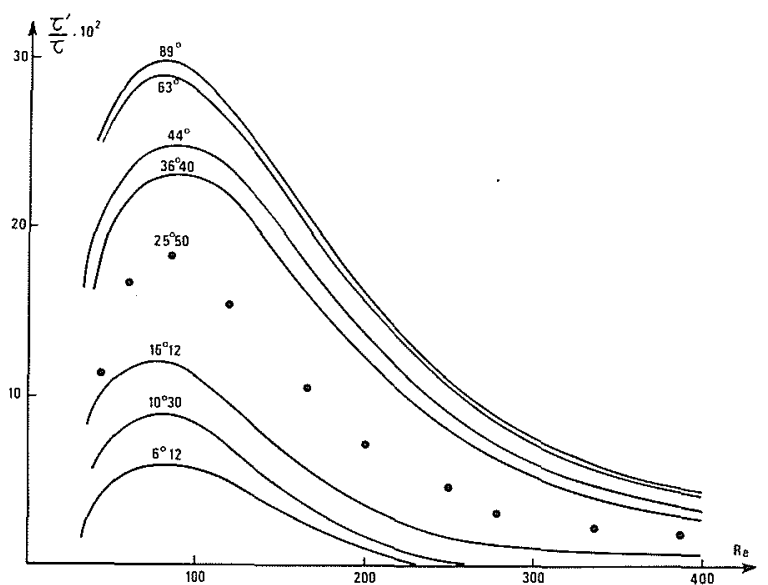

Figure 9. - Fluctuations relatives du frottement fonction de $R e$.

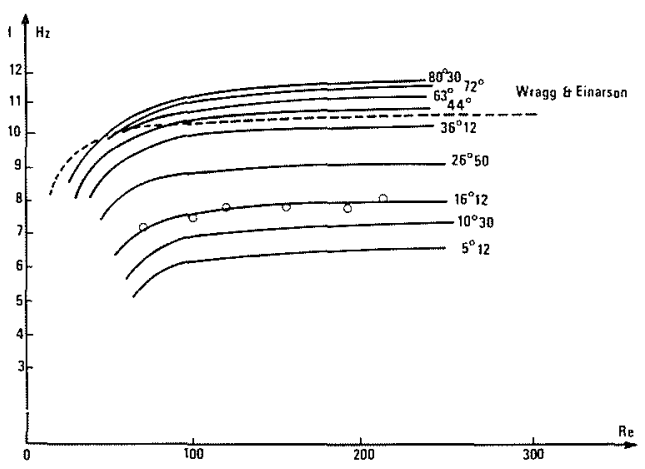

Figure 10. - Fréquences des ondes fonction du nombre de Reynolds et de l'inclinaison 
résultats expérimentaux, que la fréquence des ondes croît rapidement après leur naissance pour atteindre au-delà de $R e=100$ et jusqu'à leur disparition, une valeur pratiquement constante pour une inclinaison donnée. Son expression en fonction de l'inclinaison est alors sensiblement de la forme :

$$
f=11,5(\sin \theta)^{0,25} \text { Hertz }
$$

Les résultats obtenus dans le cas d'un tube vertical [2] sont inférieurs de $10 \%$ environ à ceux du plan vertical, mais suivent une évolution tout à fait comparable.

\section{Conclusion}

Déjà largement utilisée pour l'étude des écoulements internes [6, 7] ou externes [20] la vélocimétrie polarographique apparaît comme une technique très adaptée pour l'étude du ruissellement en film mince le long d'une paroi. Dans le présent travail correspondant au ruissellement plan, elle a permis de déterminer le frottement et d'en déduire l'épaisseur du film, de préciser le domaine des différents régimes d'écoulement, particulièrement la naissance et la croissance des ondes, puis leur dégradation progressive vers l'écoulement turbulent ainsi que la variation de leur fréquence selon le débit et l'inclinaison du plan. Les résultats obtenus qui recoupent et complètent ceux de la littérature ouvrent de vastes perspectives pour l'application de la méthode à des géométries d'écoulement plus complexes (sphère, cylindre,...) dont l'intérêt pratique est manifeste.

\section{Nomenelature des symboles}

$A$ : surface de l'électrode de mesure

$a, b$ : exposants dans l'expression du frottement

$C_{0}:$ concentration de lion actif dans la solution

$C_{f}:$ coefficient de frottement $C_{f}=\tau /\left(\rho U_{m}^{2} / 2\right)$

$D$ : coefficient de diffusion de lion actif dans la solution

$d_{0}$ : diamètre de l'électrode circulaire

$F$ : nombre de Faraday : $96500 \mathrm{C}$

$F r$ : nombre de Froude $U_{m} / \sqrt{g h}$

$f \quad$ : fréquence des ondes

$g \quad:$ attraction de la pesanteur

$h$ : épaisseur du film

$\bar{h}$ : valeur moyenne de l'épaisseur du film

$h^{\prime}$ : amplitude des fluctuations d'épaisseur du film

$h^{+}$: épaisseur adimensionnelle $:\left(h^{3} g \sin \theta\right)^{1 / 2} / \nu$

$J$ : courant limite de diffusion

$\bar{J} \quad$ : valeur moyenne du courant limite

$j$ : fluctuation du courant limite de diffusion

$J^{\prime} \quad$ : amplitude des fluctuations du courant limite

$L \quad$ : largeur de l'électrode (rectangulaire) $; l$ longueur.

$L_{e}$ : largeur équivalente de l'électrode circulaire

$N$ : nombre adimensionnel : Re $\sin \theta / F r^{2}$ $n$ : nombre d'électrons mis en jeu dans la réaction électrochimique

$Q \quad$ : débit par unité de largeur

Re : nombre de Reynolds : $Q / \nu$

$S \quad$ : gradient pariétal de vitesse

$\bar{S} \quad$ : valeur moyenne du gradient pariétal de vitesse.

$s \quad$ : fluctuations du gradient de vitesse

$u \quad$ : vitesse selon $x$

$u^{*}$ : vitesse de frottement

$u^{+}$: vitesse réduite $: u / u^{*}$

$U_{m}$ : vitesse moyenne dans le film

$x, y$ : coordonnées de plus grande pente et normale au plan

$y^{+}$: distance réduite normale au plan $y u^{*} / \nu$

$\delta_{c}$ : épaisseur de la couche limite de concentration

$\theta \quad$ : angle d'inclinaison du plan

$\rho \quad$ : masse volumique de la solution

$\mu \quad$ : viscosité dynamique de la solution

$\nu \quad$ : viscosité cinématique de la solution

$\chi$ : constante de temps de la sonde :

$$
0,911\left(L^{2} / D \bar{S}^{2}\right)^{1 / 3}
$$

$\tau \quad$ : contrainte de frottement pariétal

$\tau^{\prime}$ : amplitude des fluctuations du frottement pariétal.

\section{Références}

[1] FULFORD (G.D.) - Advances in Chemical Engineering, 1964. vol. 5. p. 151 .

[2] WRAGG (A.), EINARSSON (A.) - Chem. Eng. Science, 1970, Vol. 25, p. 67.

[3] PORTALSKI (S) - Chem. Eng. Science, 1964, Vol. 18, p. 787 et Vol. 19 , p. 575 .

[4] ISHIHARA (T.), IWAGAKI (Y.), IWASA (Y.) - Trans. Am. Soc. Civil Engrs, 1961, 126, p. 548.

[5] LEBOUCHE (M.), COGNeT (G.) - Chim. Indus. - Génie Chimique, 1967, Vol. 97, n 12, p. 2002.

[6] FORTUNA (G.), HANRATTY (T.J.) - A. I. Ch. E. Symp. Ser., 1971, Vol. 67, n 111, p. 90.

[7] COGNET (G.) - Journal de Mécanique, 1971, Vol. 10, $\mathrm{n}^{\circ} 1$, p. 65 .

[8] REISS (L.), HANRATTY (T.J.) - A. I. Ch. E., 1963, Vol. 9, p. 154 .

[9] HOPF (L.) - Ann. Physik, 1910, Vol. 32, p. 777.

[10] BINNIE (A.M.) - J. F. Mech., 1959, Vol. 5, p. 561.

[11] CAMPERGUE (D.) - Thèse de spécialité, Université de Poitiers, 1971.

[12] BOUSGARBIES (J.L.) - Thèse, Université de Poitiers, 1971.

[13] KASIMOV (B.S.), ZIGMUND (F.F.) - Inzh. Fiz. $Z h$. A kad Nauk Belorussk, 1962, Vol. 5, p. 71.

[14] NUSSELT (W.) - Ver Deut Ingr $Z, 1916,60$, p. 549.

[15] DUKLER (A.), BERGELIN (O.) - Chem. Eng. Sci., 1952 Vol. 48, p. 557.

[16] KAPITSA (S.P.) - Zh. Eksp. Teor Fiz 1949, Vol. 19 , p. 105.

[17] LEVICH (G.V.) - Physico Chemical Hydrodynamics Prentice Hall, 1962.

[18] GJEVIK (B.) - Physics of Fluids, 1970, Vol. 13, p. 1918.

[19] BROCK (R.) - J. Hydraulics Div. Am. Soc. Civil Engrs, Décembre 1970.

[20] LEBOUCHE (M.), MARTIN (M.), PY (B.) - Revue Générale de Thermique, $1972, \mathrm{n}^{\circ} 126$, p. 531 . 